\title{
As contribuições da ciência do desenvolvimento para a psicologia da saúde
}

\author{
The contributions of developmental science \\ to the health psychology
}

SimoneCerqueira-Silva ${ }^{1}$

M ariaA uxiliadora Dessen ${ }^{2}$

Áderson Luiz Costajúnior ${ }^{2}$

\footnotetext{
${ }^{1}$ Laboratório de Desenvolvimento Familiar, Instituto de Psicologia, Universidade de Brasília. Campus Darcy Ribeiro. 70910-900 Brasília DF. simonecerqueira@unb.br 2 Instituto de Psicologia, Universidade de Brasília.
}

Abstract The science of human development has been stimulating a new perspective on health research, highlighting contextual and ecological variablesthat influencetheprocess of development and condition the diagnosis, treatment and prognosis of individuals and groups. The objective of this article is to discuss about the implications of the science of human development for health psychology, specifically for the educational area aimed toward intervention in several contexts of health treatment. Another objective of this article is to critically analyze some methodological aspects os the studies in this area and point current and future tendencies at a more functional analysis of thehealth-disease process. It is expected that health professionals benefit from theargumentsand models proposed by the science of human development, designing environments that are more adequate to the psychological needs of patients and families. Key words Health psychology, Science of human development, Bioecological model, Research methods in psychology
Resumo A ciência do desenvolvimento humano vem estimulando uma nova visão sobre a pesquisa em saúde, destacando variáveis contextuais e ecológicas que influenciam o processo de desenvolvimento e condicionam o diagnóstico, o tratamento e o prognóstico de indivíduos e grupos. 0 objetivo deste artigo é discutir as implicações da ciência do desenvolvimento humano à psi cologia da saúde, área de intervenção educacional voltada aos diversos contextos de tratamento de saúde. Pretende-se, ainda, analisar criticamente alguns aspectos metodológi cos dos estudos da área eapontar tendências atuais e futuras para uma análise mais funcional do processo saúde doença. Espera-se que os profissionais de saúde se beneficiem dos argumentos e modelos propostos pela ciência do desenvolvimento humano, construindo ambientes de cuidados mais adequados às necessi dades psi cossociais de pacientes e familiares.

Palavras-chave Psicologia da saúde, Ciência do desenvolvimento humano, M odelo bioecológico, M étodos de pesquisa em psicologia 
Introdução

As últimas décadas têm registrado significativas modificações científicas etecnológicas em psicologia, propiciadas pelo crescente avanço das teorias sistêmicas, sobretudo na área do desenvolvimento humano. Os modelos sistêmicos têm demandado novas formas de gerar dados que envolvem a inter-relação entre os aspectos biológicos, sociais, culturais e históricos do desenvolvimento humano. Considerando essa evolução, acredita-se que a psicologia da saúde tem sido altamente beneficiada com as conquistas obtidas, nos últimos anos, pela ciência do desenvolvimento.

Este artigo tem o objetivo de analisar as implicações da ciência do desenvolvimento para a psicologia da saúde, destacando os aspectos teóricosemetodológicos dos estudos da área eapontando as tendências atuais e futuras no estudo sobre os processos de saúde-doença envolvendo indivíduos, grupos e populações. Na primeira seção, apresentar-se-á a psicologia da saúde enquanto campo de contribuição da psicologia, bem como os principais pressupostos metodológicos de pesquisa da área, ressaltando a relevância do uso combinado dos métodos qualitativos e quantitati vos. Na segunda seção, será discutida a perspectiva teórica da ciência do desenvolvimento, com destaque às contribuições para a compreensão do processo saúde-doença. 0 modelo bioecológico de Bronfenbrenner é apre sentado como uma das alternativas promissoras para o estudo desse processo. A terceira seção aponta algumas tendências atuais e futuras dos estudos em psicologia da saúde.

\section{Psicologia da saúde: car acterização}

A busca por variáveis psicológicas que pudessem explicar, mesmo parcialmente, a vulnerabilidade individual a determinadas doenças remonta à Grécia Clássica. No entanto, o ensino depsicologia na formação médica teve início apenas no começo do século XX, nos Estados Unidos da América, ao mesmo tempo em que ocorriam as primeiras inserções de psicólogos em hospitais gerai ${ }^{1,2}$. No Brasil, os primeiros serviços de psicologia no hospital foram instalados na década de cinquenta, no H ospital das Clínicas da Faculdade de M edicina da Universidade de São Paulo².

Ao longo do século $X X$, o reconhecimento científico de que fatores psicológicos interferem sobre a etiologia de doenças somáticas e a de- monstração de que a prestação de serviços de assistência à saúde inclui um complexo processo de interações sociais entre indivíduos dispostos hierárquica e funcionalmente subsidiaram um movimento crescente de questionamento à orientação biomédica da assistência à saúde e de ampliação da perspectiva social da medicina. Tais questionamentos levaram ao desenvolvimento de modelos de saúde que priorizam a educação e a atenção integral ao indivíduo - modelos biopsicossociais - em detrimento de modelos médicos tradicionais - modelos biomédicos - ainda vigentes na mai or parte dos sistemas de saúde ${ }^{1,3,4} \mathrm{e}$ à criação da psicologia da saúde, Divisão 38 da American Psychological Association, em 1978.

A psicologia da saúde, fundamentada no modelo biopsicossocial, representa um rompimento com o modelo linear de saúde, de causa e efeito, centrado em eventos biológicos como causadores de doença na tecnologia médico-farmacológica como principal opção resolutiva. Fatores biológicos, psicológicos esociais são considerados como integrados e inter-relacionados ao processo de saúde-doença, constituindo importantes indicadores de como indivíduos e grupos enfrentam processos de doença eaderem, ou não, a prescrições de tratamento médico $0^{3,5}$.

Enquanto um campo decontribuição específica da psicologia, científica e profissional, a psicologia da saúde prioriza a promoção ea manutenção da saúde, bem como a prevenção e o tratamento das doenças. Para isso, busca a identificação de relações funcionais entre os fatores psicossociais (por exemplo: idade, gênero, status socioeconômico, hábitos devida, raça, redesocial deapoio, comportamentos, etc.) e a etiologia, o diagnóstico e 0 prognóstico de doenças e disfunções ${ }^{1,4}$.

Devemos lembrar que a própria Organização Mundial da Saúde, desde $1970^{6}$, quando estabeleceu os princípios fundamentais da saúde (considerar a pessoa dentro do seu contexto sociocultural; investigar a exposição da pessoa a fatores de risco e vulnerabilidades; avaliar os fatores protetores do desenvolvimento da pessoa; promover ações em todos os níveis de aten ção à saúde e priorizar a educação para a saúde), já apontava as vantagens de modelos de promoção e atenção integral à saúde.

Destaca-se, ainda, que a psicologia da saúde se caracteriza pelo agrupamento de intervenções educacionais que podem ser aplicadas a diferentes problemas deum sistema de saúde, incluindo usuários, recursos humanos, instituições e políticas de saúde. Trata-se de uma área em plena expansão, com crescente reconhecimento acadê- 
mico, profissional e de associações científicas, tanto brasileiras quanto estrangeiras. Uma das implicações deste fato éo crescimento do número de periódicos especializados e de psicólogos inseridos em serviços de saúde pública e privada. Atualmente, vários temas têm sido investigados, dentre os quais merecem destaque a adesão ao tratamento (por exemplo, os estudos de Arru$\mathrm{da}^{7}$, Barletta ${ }^{8}$, Ferreira ${ }^{9}$ e de Tanesi et al. ${ }^{10}$ ); as estratégias deenfrentamento - coping (por exemplo, os estudos de Koenig ${ }^{11}$, Lorencetti e Simonetti ${ }^{12}$; Panzini e Bandeira13; Pereira e Araúijo ${ }^{14}$; Santos eAlves Júnior ${ }^{15}$; Santos et al. ${ }^{16}$ e deSeidl et al. ${ }^{17}$ ); indicadores dequalidade devida ( por exemplo, os estudos de $M$ inayo et al. ${ }^{18}$ e Ravagnani et al. ${ }^{19}$ ); aspectos comportamentais e contextuais das doenças (por exemplo, o estudo de Flores ${ }^{20}$ ) e educação para a saúde (por exemplo, os estudos de Ferreira ${ }^{21}$ e de Jenkins ${ }^{22}$ ).

Entretanto, épossível apontar que os estudos carecem de um delineamento de pesquisa mais sistemático para a obtenção de informações funcional mente rel evantes das inter- rel ações da pessoa em desenvolvimento e o seu ambiente de cuidados. A seguir, apresentamos um panorama da situação atual e uma proposta do uso combinado dos métodos qualitativos e quantitativos em investigações do processo saúde-doença.

M etodologia de pesquisa nos estudos do processo saúde-doença:

necessidade do uso combinado de métodos quantitativos e qualitativos

É possível constatar que há uma supremacia do uso de métodos quantitativos nos estudos sobre processo saúde-doença. Em levantamento realizado em uma das principais revistas de saúde no Brasil, Cerqueira-Silva et al. ${ }^{23}$ constataram que no período de janeiro de 2001 a outubro de 2007, das 933 publicações, 122 tratavam dequestões referentes à saúde da criança e do adolescente e, dessas, a maioria (91\%) empregou a metodologia quantitativa, sobretudo a estatística inferencial. As autoras concluíram que, apesar da maior parte dos artigosconsiderarem variáveis biopsicossociais, notase que al gumas dessas variáveis foram consideradas apenas nas discussões dos artigos e, não propriamente, na coleta e análise dos dados.

As pesquisas na saúde, em sua maioria, utilizam o método quantitativo devido à tradição do paradigma positivista e sua preocupação com a generalização dos resultados, o queinevitavelmenteremeteaos testes estatísticosea um maior núme- ro de participantes de pesquisa ${ }^{24}$. Nesse modelo de pesquisa, as relações causais entre algumas doenças e uma determinada condição biológica deuma população têm sido propostas como principal elemento explicativo. Esta tendência, nem sempre apropriada, se deve à grande influência da formação e do contexto médico biológico.

Grady e Wallston ${ }^{6}$, por exemplo, descrevem os passos necessários para o delineamento de uma pesquisa na área da saúde, em que ficam evidenciadas tanto a generalização dos resultados quanto a precisão no controle e medida das variáveis. Tais princípios são típicos das pesquisas queempregam delineamentos experimentais, que por si só não satisfazem plenamente as exigências do estudo de processos de desenvolvimento humano.

Contudo, éimportante ressaltar que a metodologia quantitativa constitui um importante recurso de investigação do processo saúde-doença, uma vez que propicia compreender relações entrevariáveis, pressupondo descrição, predição e controle ${ }^{25}$. Portanto, este tipo de método reúne, registra e anal isa dados numéricos; utiliza critérios estatísticos de representatividade amostral; busca identificar e quantificar as diferenças que existem num mesmo segmento efornece resultados que permitem uma conclusão e compreensão geral do fenômeno estudado ${ }^{26}$.

Em consonância com essas contribuições do método quantitativo, a maioria dos estudos tem empregado análises estatísticas inferenciais, próprias desse método (por exemplo, Assunção et al. ${ }^{27}$; Ferriolli et al. ${ }^{28}$; Leal et al. ${ }^{29} \mathrm{eM}$ acedo et al.$^{30}$ ). Estes estudos mostram que uma investigação, em uma amostra representativa da população, que visa identificar associações entre diferentes variáveis ou a prevalência de um determinado fator na amostra estudada, em muito se beneficia dos recursos da estatística inferencial.

A pesar da predominância dos estudos quantitativos, tem crescido o interesse e o uso de métodos qualitativos em saúde. 0 reconhecimento da importância defatores sociais, políticos eeconômicos sobre as condições de saúde e doença de indivíduos e grupos, tais como fumo, violência, obesidade, etc., tem apontado para a necessidade de se compreender, mais precisamente, 0 papel das variáveis biopsicossociais, o que tem exigido delineamentos metodológicos mais sofisticados depesquisa, não exclusivamente quantitativos. Em outras palavras, para estudar a complexidade das interações humanas, considerando a influência dos fatores contextuais, faz-se necessário que os pesquisadores conduzam pes- 
quisas no contexto de vida natural das pessoas em desenvolvimento, o que requer, também, 0 uso de métodos qualitativos ${ }^{24}$.

Confirmando a importância do método qualitativo de pesquisa em psicologia da saúde, Stiles $^{31}$ afirma que esta éa melhor maneira de estudar a experiência humana e seus significados e que esse tipo de análise favorece os estudos de caso ou de pequenos grupos, em contraposição aos estudos que utilizam amostras representativas. 0 autor destaca, ainda, que o método quantitativo, baseado no modelo linear de causa e efeito, em correlações e regressões estatísticas, tem limitações para tratar de questões não lineares, como os processos de interações humanas.

Em geral, o método qualitativo consiste em uma pesquisa naturalística, tem dados descritivos, enfatiza o processo, é indutivo e considera essencial a questão da significação ${ }^{26,32}$. Além disso, caracteriza-se como uma abordagem multimetodológica quanto ao seu foco e interpretativa, pois busca dar sentido ou interpretar os fenômenos em termos das significações queas pessoas trazem para eles ${ }^{33}$. As características da pessoa ou das suas interações podem ser avaliadas, por exemplo, a partir de um questionário e/ou de uma entrevista, por sessões de observação de comportamentos interpessoais, em diferentes situações coti dianas ou por escalas ou inventários.

0 estudo de Darbyshir ${ }^{34}$ ilustra a aplicação do método qualitativo em psicologia da saúde, tendo como fundamentação teórica a abordagem fenomenológica. 0 objetivo desse estudo foi investigar a percepção dos genitores quanto à recuperação de crian ças hospitalizadas. Para Darbyshire, o seu estudo exploratório, o qual não tinha a intenção de generalizar os resultados, apresentou dados ricos e detalhados quanto às experiências "daquelas mães e naquel e contexto". O utros estudos, mais recentes ${ }^{34-38}$,também têm utilizado 0 método qualitativo para compreender diversos fenômenos relacionados ao processo saúde-doença, tais como questões referentes ao desenvolvimento de estratégias de enfrentamento, à depressão, ao alcoolismo, às relações de gênero e paternidade e ao consumo de drogas ilícitas.

A pesar das contribuições de ambos os métodos para a compreensão do processo saúde-doença, a oposição entre a qualidade e a quantidade tem sido uma constante nos estudos da psicologia da saúde, embora esses métodos de coleta e análise de dados sejam inter-relacionados e complementares ${ }^{39,40}$. Além disso, constata-se que as investigações deixam a desejar quanto ao uso deabordagem multimetodológica; à coleta deda- dos em ambiente natural e em diferentes momentos de experiência; ao acesso aos diferentes níveis do ambiente ecológico da pessoa em desenvolvimento e ao acesso aos processos proximais que promovem a saúde e/ou doença ${ }^{23}$.

Uma das alternativas para a pesquisa na psicologia da saúde, em consonância com o modelo biopsicossocial e com o uso combinado dos mé todos quantitativos equalitativos, é empregar os pressupostos norteadores da ciência do desenvolvimento que têm sido recentemente difundidos no Brasil|4. A seguir, serão apresentadas algumas questões teóricas e conceituais referentes ao desenvolvimento humano, destacando, em especial, as suas contribuições para a compreensão do processo saúde-doença. 0 modelo bioecológico de Bronfenbrenner é proposto como uma das alternativas a ser implementada na pesquisa em psicologia da saúde.

\section{0 desenvolvimento humano e suas contribuições para a compreensão do processo saúde-doença}

No decorrer da segunda metade do século XX, vimos ocorrer inúmeras modificações nas ciências em geral e, em especial, o surgimento de uma ciência para o estudo do desenvolvimento: a ciência do desenvolvimento ${ }^{42}$. Esta ciência tem sido considerada um caminho promissor para o estudo científico do curso de vida, cujo processo é marcado por dinâmicas inter-relações entre os sistemas que existem dentro e fora da pessoa.

A pessoa, na interação com o ambiente, éconsiderada um organismo ativo, que influencia eé influenciada por ele e depende das interações recíprocas entre os seus subsistemas (cognitivo, emocional, fisiológico, perceptual e neurobiológico) que, por sua vez, também se influenciam mutuamente. Portanto, o desenvolvimento do indivíduo ocorre demodo organizado hierarquicamenteem múltiplos níveis quepodem influenciar-se reciprocamente: dos genes ao citoplasma, célula, sistema de órgãos, organismo, comportamentos e meio ambiente. 0 trânsito dessas influências é bidirecional. Logo, os genes formam uma parte integral do sistema e sua atividade é afetada pelos eventos dos seus outros níveis, incluindo 0 ambiente e o organismo ${ }^{43}$. Por exemplo, uma baixa produção hormonal pode ser afetada pelos eventos ambientais tais como a luz, duração do dia, nutrição e comportamentos.

Nesse sentido, o desenvolvimento humano tem sido concebido como (a) um processo con- 
tínuo no tempo; (b) em permanente mudança; (c) progressivamente mais complexo e (d) causado por atividades, experiências e coações desenvolvidas na interação entre a pessoa e seu ambiente ${ }^{44-47}$.

Coações são as tensões criadas nos níveis estrutural e funcional do organismo quando este se depara com circunstâncias novas ou adversas à manutenção do equilíbrio ${ }^{44}$. Para Gottlieb ${ }^{48}$, o conceito maisfrequentemente utilizado para designar as coações no nível do funcionamento do organismo são as experiências, que podem ser tanto favoráveis quanto desfavoráveis ao desenvolvimento humano. Por exemplo, as doenças podem representar uma experiência adversa para a pessoa em desenvolvimento, provocando de sequilíbriose crisestanto em seu organismo quanto nos seus diferentes ambientes de vida.

0 desenvolvimento humano, assim concebido, passou a ser compreendido como multideterminado, isto é, de causalidade sistêmica. Em outras palavras, passou a constituir um processo ao mesmo tempo universal e individual, que influencia eéinfluenciado por contextos externos ambiente físico esocial - e por contextosinternos - o próprio organismo histórico e biológico -, em dimensões de tempo e espaço específicos ${ }^{49}$.

$N$ esse sentido, éuma futilidade tanto o debate dos geneticistas comportamentais so brea contraposição entre biologia e ambiente, quanto a resistência dos defensores do contextualismo ao ignorarem as diferenças biológicas entre os humanos ${ }^{50}$. A não separação do contexto e organismo garantea identificação e compreensão dos determinantes do comportamento humano, e a inter-relação entre genótipo efenótipo exemplifica a bidirecionalidade que caracteriza o desenvolvimento, incluindo tanto fatores biológicos quanto sociais.

Compartilhando dessa mesma concepção, Dal-Farra e Prates ${ }^{51}$ consideram que os estudos sobre o desenvolvimento humano demonstram que tanto os fatores ambientais quanto os fatores genéticos são fundamentais para a estruturação do ser humano. Os genes influenciam o comportamento, mas não o determinam, e as tendências genéticas na construção das doençastambém são probabilísticas e não deterministas, ge rando um mosaico de possibilidades do desenvolvimento humano.

Neste contexto, as pesquisas futuras sobre a relação entre genética e ambiente no desenvolvimento humano devem ir além de simplesmente perguntar "se" e "quanto" os fatores genéticos influenciam o comportamento, sendo necessá- rio identificar como o genótipo torna-se fenóti$\mathrm{po}^{52}$. Portanto, as pesquisas futuras nessa área devem não somente esclarecer como a interação gene-ambiente modela o desenvolvimento humano, mas também fornecer informações sobre as características ambientais e hereditárias que favorecem ou dificultam as competências funcionais ${ }^{49}$. Em outras palavras, isto significa investigar as diferenças individuais que podem ser atribuídas ao potencial genético eàs conexões com 0 ambiente.

Assim, uma das alternativas para se compreender melhor o processo saúde-doença é adotar, na prática da pesquisa, os pressupostos da ciência do desenvolvimento. A seguir, apresentamos os argumentos necessários para a investigação desse processo com base na ciência do desenvolvimento.

O processo saúde-doença sob a ótica da ciência do desenvol vimento

A saúde e a doença podem ser compreendidas como duas diferentes modalidades do desenvolvimento. Na primeira, o processo de desenvolvimento humano caracteriza-se como um estado de excelência em todas as suas condições (físicas, biológicas, emocionais, bioquímicas, sociais, etc.). $\mathrm{N}$ a segunda, esse mesmo processo se constitui de modo desequilibrado, disfuncional, com todas essas mesmas condições em desarmonia. Estes dois estados do desenvolvimento variam e se diferenciam considerando a ampla gama de fatores que podem interferir nessa configuração, sejam variáveis externas ou internas à pessoa.

Tanto a saúde quanto a doença são processos multideterminados por aspectos intra e inter sujeitos, com origem e evolução perfazendo uma trajetória probabilística. Isto significa dizer que seu resultado depende de um conjunto de fatores (do nível micro ao nível macro), queinterconectados se influenciam mutuamente, podendo mudar o rumo do desenvolvimento em qualquer fase da vida ${ }^{43}$. Assim, podem ser criados instrumentos promocionais de saúde epreventivos à instalação de patologias crônicas ao longo da vida das pessoas.

Na perspectiva do curso devida, assumida pela ciência do desenvolvimento, fica difícil dizer o que seja um padrão normal ou patológic ${ }^{53}$. 0 curso de vida representa uma orientação teórica para o estudo do desenvolvimento humano que incorpora distinções temporais, contextuais e processuais. 0 curso de vida envolve o indivíduo e a 
sociedade; maturação, crescimento, forças sociais e contextos socioculturais em diferentes níveis ${ }^{54}$.

Assim, o processo saúde-doença se caracteriza como um processo em desenvolvimento, construído a partir do interjogo entre o fenótipo e o genótipo. Nessa teia de relações, os processos proximais (atividades realizadas) funcionam como coações, podendo desencadear avanços ou retrocessos, equilíbrios ou desequilíbrios e, consequentemente, transtornos ou doenças. Assim, a doença ou a saúde podem ser compreendidas como contextuais, isto é, dependem do ambiente no qual a pessoa vive. Podem, ainda, ser compreendidas como dinâmicas, uma vez que mudam de acordo com o curso de vida.

Considerando essa natureza dinâmica do de senvolvimento humano, no processo de saúde doença, a pessoa é ativa no seu desenvolvimento e, consequentemente, suas escol has podem ter um papel substancial no curso desse processo, incluindo o curso de doenças ${ }^{55}$. Esta concepção difere daquela do modelo tradicional de pesquisa.

Tendo em vista essa noção do processo saúde-doença na perspectiva da ciência do desenvolvimento, os diagnósticos e prognósticos levam em consideração uma variedade de fatores tanto internos quanto externos da pessoa, em suas avaliações e tratamentos propostos. Somado a isso, a dimensão probabilística do curso de vida desfaz as previsões deterministas, comumente realizadas em prognósticos médicos restritos a variáveis biológicas, e, sobretudo, real ça a importância das estratégias de intervenção e de decisão da pessoa em desenvolvimento, bem como o papel dos profissionais de saúde como cuidadores da pessoa em desenvolvimento etemporariamente exposta a contextos de tratamento médico.

No que tange à pesquisa na perspectiva da ciência do desenvolvimento, o processo de saúde-doença deve ser investigado de modo multidisciplinar einterdisciplinar $45,53,56,57$, incorporando os diferentes níveis do ambiente ecológico, nos quais a pessoa se desenvolve. I sto requer contribuições da interface de diversas disciplinas científicas tradicionais: biologia, psicologia, fisiologia, neuropsicologia, psicologia social, sociologia, antropologia. N essa perspectiva, a ênfase nos estudos desses processos está nas múltiplas causas e nos múltiplos níveis de análises, bem como nas interações, no papel da família e na dinâmica dos sistemas mais amplos, como a política ${ }^{55}$. Portanto, o modelo bioecológico proposto por Bronfenbrenner ${ }^{58,59}$ constitui uma das alternativas promissoras para investigação do processo de saúde-doença.
O modelo bioecológico deBronfenbrenner: em busca de uma alternativa para o estudo do processo saúde-doença

Bronfenbrenner ${ }^{44,60}$, descontente com as limitações científicas nas abordagens que prevaleciam para a pesquisa em desenvolvimento humano, e insatisfeito com a configuração da psicologia do desenvolvimento atémeados da década de setenta (século XX), propôsum modelo para orientar pesquisas em desenvolvimento humano, intitulado modelo bioecológico de desenvolvimento humano. Sua principal crítica foi relativa às pesquisas conduzidas pela psicologia do desenvolvimento, queeram sobreum comportamento estranho, em situações estranhas, com adultos estranhos e em breves períodos de tempo. Este modelo reorientou a tradicional concepção da psicologia de então, que atomizava as funções psicológicas e dava aos processos psicológicos uma conotação demasiado individualista ou intimista ${ }^{61}$.

D efendendo a importância dos planejamentos de pesquisas em ambientes naturais e sustentando que, para se entender o desenvolvimento humano, deve-se considerar o sistema ecológico inteiro no qual a pessoa está inserida, Bronfenbrenner ${ }^{44}$ se interessou pelos processos e pelas condições que governam o desenvolvimento humano nos ambientes naturais, nos quais os seres humanos vivem. Nessa perspectiva, 0 ambiente significa o contexto social maior e não só o local imediato em que a pessoa se desenvolve. Portanto, ele criticou a visão de ambiente como sendo 0 local concreto que contém a pessoa, que predominava nas pesquisas realizadas na época, e enfatizou a importância de se ampliar esse conceito.

Com base nesse conceito ampliado de ambiente, Bronfenbrenner ${ }^{44,60}$ destaca a necessidade de ir além da observação direta do comportamento deuma ou mais pessoas no mesmo local para compreender o desenvolvimento humano. Além disso, afirma ser necessário levar em consideração aspectos além do ambiente imediato que contém a pessoa, para incluir outros sistemas macrossociais ao longo do tempo histórico. Portanto, a ecologia do desenvolvimento humano consiste no estudo científico das acomodações progressivas e mútuas entre o crescente organismo humano eo ambiente imediato em que ele vive, ao longo do curso de vida ${ }^{44}$. Em outras palavras, um estudo fundamentado no modelo bioecológico de Bronfenbrenner permiteanalisar as variações do processo edo produto do desenvolvimento enquanto função conjunta das características da pessoa e do ambiente em um determinado período histórico62. 
Nessesentido, 0 ambiente ecológico éconce bido como um arranjo de estruturas concêntricas inseridas umas nas outras. Inicialmente, como centro dessas estruturas, está o microssistema, queé definido como o complexo de relações entre a pessoa em desenvolvimento e o ambiente imediato que a contém (casa, escola, trabalho). $N$ esses locais, as pessoas se engajam em atividades e papéis específicos, em determinados períodos do tempo, que são vivenciados face a face com filhos, pais, professores, alunos e profissionais, dentre outros. 0 mesossistema, segundo componente do modelo, refere-se ao conjunto de microssistemas e às inter-relações entre esses locais imediatos que contem a pessoa, ou seja, entrea escola ea família, entrelocal detrabalho e a família. Esses locais mudam ao longo do tempo, uma vez que a pessoa em desenvolvimento passa a fazer parte de diferentes locais.

Já o exossistema é uma extensão do mesossistema, envolvendo outras estruturas sociais, formais e informais, que não contém a pessoa em desenvolvimento, mas que influenciam o seu desenvolvimento. Em se tratando de filhos pequenos, o trabalho dos pais constitui um exemplo de exossistema. Por outro lado, o macrossistema abrange os sistemas de valores e crenças que permeiam a existência e a evolução das diversas culturas e subculturas, tais como a economia, políticas sociais, educacionais e legais $s^{44}$.

Além disso, Bronfenbrenner e seus colaboradores ${ }^{63,64}$ ressaltaram a necessidade de olhar a pessoa em desenvolvimento considerando o fator tempo, que ele denominou de cronossistema. Este conceito implica que a passagem do "tempo" não é um atributo meramente do ser humano em crescimento, mas é também uma propriedade do ambiente. Isto é, as mudanças ou continuidades ao longo do tempo ocorrem não só nas características da pessoa, mas também no ambiente no qual ela vive, tais como na estrutura familiar, no status socioeconômico, no emprego, no lugar de residência ou no grau de habilidade na vida diária ${ }^{57}$.

Com base nos diferentes níveis desse ambiente, do micro ao macrossistema, Bronfenbrenner ${ }^{44}$ propõe que um estudo sobre o desenvolvimento humano, para ser válido ecologicamente, precisa demonstrar compatibilidade entreo problema que se propõe a investigar e o local para essa investigação. Isto significa queum ambiente natural não é, necessariamente, válido para todos os estudos do desenvolvimento humano. Além disso, um experimento ecológico, na con- cepção deBronfenbrenner, deveinvestigar o contexto de desenvolvimento, mostrando os processos recíprocos, ou seja, o efeito de $A$ sobre $B$ e também o efeito de $B$ sobre $A$. Outro destaque dado por ele refere-se ao efeito de segunda ordem - influência indireta de uma terceira pessoa ou dos efeitos indiretos de fatores físicos no desenvolvimento do indivíduo. Em geral, os ruídos, as informações da tel evisão, a disposição do mobiliário, as cores em um ambiente constituem exemplos de efeitos de segunda ordem.

Outra característica desse model o consistena importância de se estudar o desenvolvimento humano em períodos denominados de transições ecológicas, normativas ou não normativas, que são as mudanças na vida das pessoas diante de papéis e atividades diferentes que são desempenhados ao longo de sua vida. Por exemplo, o deslocamento deum bebêrecém-nascido do hospital para casa, a entrada de uma criança na préescola ou quando uma pessoa muda de emprego ou torna-se esposa, mãe ou irmã, são mudanças na vida de um membro familiar que podem alterar todo o sistema de relações familiares, e são consideradas normativas ${ }^{44}$. As transições ecológicas não normativas são consideradas comuns no contexto da saúde, isto é, em condições adversas ou atípicas de desenvolvimento: a própria hospitalização, a comunicação de um diagnóstico adverso ou, ainda, a exposição da pessoa a procedimentos médicos invasivos.

Deacordo com os princípios do modelo bioecológico de Bronfenbrenner ${ }^{65}$, um delineamento de pesquisa que tem por objetivo investigar as características psicológicas da pessoa e seu desenvolvimento deve permitir comparações sistemáticas e interpretações de avaliações em diferentes contextos. Por exemplo, em se tratando de uma avaliação da competência cognitiva e/ou socioemocional de um indivíduo ou grupo, esta deveria ser interpretada à luz da cultura ou subcultura na qual a pessoa vive. Além disso, tais comparações devem ser feitas por observadores que diferem em seu papel eem sua relação com a pessoa foco do estudo (pais, professores, supervisores e pesquisadores treinados), e, ao mesmo tempo, pela própria pessoa, cuja percepção deve ser levada em conta.

Em síntese, este modelo destaca a importância de se pesquisar os processos de interação entre a pessoa e 0 ambiente, as características da pessoa, as características relativas ao tempo histórico, cultural e social, e por fim, as características do contexto - ambientefísico, social ecultural. 
Considerações finais: ten dências atuais efuturas nos estudos da psi cologia da saúde

Uma proposta de estudo que visa investigar a saúde e a doença pode ter como eixos teóricos norteadores, sobretudo, a ciência do desenvolvimento e, em especial, o modelo bioecológico de Bronfenbrenner. Essa ciência possibilita que os processos de desenvolvimento sejam considerados em sua natureza sistêmica e inter-relaciona$\mathrm{da}$, oferecendo conceitos e propondo medidas que permitem descrever a pessoa em seu contexto, tempo e espaço específicos ${ }^{66}$.

De acordo com as concepções da ciência do desenvolvimento, o processo de saúde-doença é considerado como um fenômeno social, dinâmico e cultural. A proposta de aplicação do modelo bioecológico de Bronfenbrenner na área da psicologia da saúde, particularmente para a investigação do processo de saúde-doença, poderá abrir um campo promissor para responder questões centrais dainterfaceentresaúdee desenvolvimento humano. 0 estudo de tais questões exige a adoção de modelos que possam capturar a complexidade e a inter-relação típicas de fenômenos sistêmicos e holísticos, pouco explorados até o presente. Não resta dúvida que a tarefa é desafiadora, especialmenteporqueimplica aplicar o pensamento sistêmico na prática de pesquisa.

Segundo Nicassio et al. ${ }^{4}$, os avanços da psicologia da saúde apontam para algumas recomendações futuras quanto à pesquisa, aplicações clínicas e políticas públicas. Em primeiro lugar, eles ressaltam a importância de se adotar abordagens interdisciplinares na pesquisa em saúdee defendem que as intervenções psicológicas devem ser teórica eempiricamentefundamentadas, além de específicas e, consequentemente, diferenciadas ou sensíveis ao gênero, cultura, status socioeconômico e toda variedade de fatores contextuais. Para eles, os profissionais devem considerar os princípios éticos subjacentes às intervenções e que a pesquisa e a prática clínica estão inter-relacionadas. Estas recomendações, sem sombra de dúvida, são consistentes com a perspectiva bioecológica.

Suls e Hothman ${ }^{5}$, por sua vez, também ressaltam a importância de se estudar as diferenças culturais e étnicas no desenvolvimento das doenças e de coletar informações a respeito de todos osfatores quecontribuem para o desenvolvimento do ser humano, ao longo do tempo. Segundo esses autores, os pesquisadores não têm avaliado todos os fatores do desenvolvimento humano; eles constataram, por exemplo, que na revista $\mathrm{H}$ ealth Psychology, 94\% dos estudos publicados entre 2001 e2002 avaliavam apenas variáveis psicológicas e metade avaliou, além de variáveis psicológicas, variáveis sociais e biológicas.

Para que a psicologia da saúde possa dar uma contribuição efetiva, relevantetanto do ponto de vista social quanto científico, faz-se necessário que se repense o model o de comportamento humano relacionado ao processo de saúde-doença esereexamineas necessidades pedagógicas e práticas do campo multidisciplinar e interdisciplinar ${ }^{67}$. Além disso, deve-se partir de uma abordagem multinível, pois a melhoria das condições de saúde depende deestratégias queenvolvem múltiplos conhecimentos: da saúde individual, das relações sociais, da legislação e dos fatores ambientais. Em se tratando especificamente dos métodos de pesquisa na psicologia da saúde, é importante destacar o que foi requerido por $\mathrm{He}$ pworth ${ }^{67}$ : um pluralismo metodológico para trabalhar com idéias interdisciplinares e uma combinação de abordagens de pesquisa. N esse sentido, o autor considera necessário que seja sel ecionado um delineamento de pesquisa apropriado para tratar das questões específicas de saúde e que esses possam integrar métodos quantitativos e qualitativos.

Portanto, faz-se necessário que a psicologia da saúde invista em pesquisas a respeito dos processos de desenvolvimento humano, queanalisee compreenda os diferentes contextos de vida, incluindo a diversidade de trajetórias do indivíduo no curso de vida. Reforçar o compromisso e seu comprometimento com o ambiente social, com as relações interpessoais, superando 0 atendimento clínico curativo, deveriaser prioritário naagenda da área. Isto requer conhecer a população a ser atendida, seus valores, crenças, práticas, bem como suas dificuldades e preferências, e, consequentemente, relacionar model os de saúde-doença com distribuição geográfica, nível socioeconômico, perfil epidemiológico, gênero, idadeeetnia.

N este contexto, a pesquisa em psicologia da saúdeque se propõea investigar, sistematicamente, os múltiplos fatores envolvidos no processo de saúde-doença, pode usufruir dos fundamentos teóricos da ciência do desenvolvimento e, especialmente, do modelo bioecológico de Bronfenbrenner. Ambos se apresentam como uma alternativa promissora e coerente filosoficamente com as premissas da psicologia da saúde. 


\section{Colaboradores}

SCerqueira-Silva participou da concepção do artigo e revisões posteriores; M A Dessen realizou revisões críticas e reformulações do artigo após elaboração inicial; AL Costajúnior foi responsável pela revisão e aprovação da versão final a ser publicada.

\section{Referências}

1. Ogden J. Psicologia da saúde. Lisboa: Climepsi; 1999.

2. De M arco M A. Psicologia da Saúde. In: De M arco M A, organizador. A face humana da medicina. São Paulo: Casa do Psicólogo; 2003. p. 71-76.

3. Matarazzo JD. Behavioral health and behavioral medicine: Frontiers for a new health psychology. Am Psychol 1980; 35(9):807-817.

4. Nicassio PM, M eyerowitz BE, Kerns RD. The future of health psychology interventions. Health Psychol 2004; 23(2):132-137.

5. Suls J, Hothman A. Evolution of the biopsychosocial model: Prospects and challenges for health psychology. Health Psychol 2004; 23(2):119-125.

6. Grady KE, Wallston BS. Research in health care settings. N ewbury Park: Sage Publications; 1998.

7. Arruda PM. Exigências para adesão ao tratamento pediátrico de febre reumática e diabetes M elitus tipo I e estratégias de enfrentamento do cuidador [tese]. Brasília (DF): Universidade de Brasília; 2002.

8. Barletta JB. Treinando respostas de adesão ao tratamento em portadores de aids hospitalizados: um estudo exploratório [dissertação]. Brasília (DF): Universidade de Brasília; 2003.

9. Ferreira EAP. Adesão ao tratamento em portadores de diabetes: efeitos de um treino em análise de contingências sobre comportamentos de autocuidado [dissertação]. Brasília (DF): Universidade de Brasília; 2001.

10. Tanesi PHV, Yazigi L, Fiore M LM, Pitta JCN. Adesão ao tratamento clínico no transtorno de personalidade borderline. Estud. psicol. 2007; 12(1):71-78.

11. Koenig HG. Religião, espiritualidade e transtornos psicóticos. Rev. Psiq. Clín. 2007; 34(1):95-104.

12. Lorencetti A, Simonetti JP. As estratégias de enfrentamento de pacientes durante 0 tratamento de radioterapia. Rev. Latino-am Enfermagem 2005; 13(6):944950.

13. Panzini RG, Bandeira DR. Coping (enfrentamento) religioso/espiritual. Rev. Psiq. Clín. 2007; 34(1):126135.

14. Pereira MEM SM, Araújo TCCF. Enfrentamento e reabilitação de portadores de lesão medular e seus cuidadores. PSICO 2006: 37(1):37-45.

15. Santos AF, Alves Júnior $A$. Estresse e estratégias de enfrentamento em mestrandos de ciências da saúde. Psicol. Reflex. Crit. 2007; 20(1):104-113.

16. Santos AF, Santos LA, M elo DO, Alves Júnior A. Estresse e estratégias de enfrentamento em pacientes que serão submetidos à cirurgia de colecistectomia. Interação em Psicol 2006; 10(1):63-73.

17. Seidl EM F, Rossi WS, Viana KF, Meneses AK, M eireles $\mathrm{E}$. Crianças e adolescentes vivendo com HIV/ Aids e suas famílias: aspectos psicossociais e enfrentamento. Psic. Teor. e Pesq. 2005; 21(3):279-288.

18. M inayo M CS, Hartz ZMA, Buss PM. Qualidade de vida e saúde: um debate necessário. Cien Saude Colet 2000; 5(1):7-18.

19. Ravagnani LMB, Domingos NAM, M iyazaki M COS. Qualidade de vida e estratégias de enfrentamento em pacientes submetidos a transplante renal. Estud. psicol. 2001; 12(2):177-184.

20. Flores AM N. Diagnósticos em processo: aspectos comportamentais e contextuais das cefaléias primárias [dissertação]. Brasília (DF): Universidade de Brasília; 2005. 
21. Ferreira RS. Efeitos da apresentação sistematizada de um manual educativo para pais de crianças com leucemia [dissertação]. Brasília (DF): Universidade de Brasília; 2005.

22. Jenkins $C D$. Construindo uma saúde melhor. Porto Alegre: Artmed/OPAS; 2007.

23. Cerqueira-Silva S, Senna SRCM, Alves FB. Saúde da criança e do adolescente. Manuscrito não publicado; 2007.

24. Jack SM. Utility of qualitative research findings in evidence-based public health practice. Public $\mathrm{H}$ ealth N urs 2006; 23(3):277-283.

25. Abbad G, Torres CV. Regressão múltipla stepwise e hierárquica em psicologia organizacional: aplicações, problemas e soluções. Estud. psicol. 2002; 7 (número especial):19-29.

26. Turato ER. M étodos quantitativos e qualitativos na área da saúde: definições, diferenças e seus objetos de pesquisa. Rev. Saude Publica 2005; 39(3):507-514.

27. Assunção MCF, Santos IS, Barros AJD, Gigante DP, Victora CG. Anemia em menores de seis anos: estudo de base populacional em Pelotas, RS. Rev. Saude Publica 2007; 41(3):328-335.

28. Ferriolli SHT, Marturano EM, Puntel LP. Contexto familiar e problemas de saúde mental infantil no programa saúde da família. Rev. Saude Publica 2007; 41(2):251-259.

29. Leal MC, Gama SGN, Cunha CB. Desigualdades sociodemográficas e suas conseqüências sobre 0 peso do recém-nascido. Rev. Saude Publica 2006; 40(3):466-473.

30. Macedo SEC, M enezes AM B, Albernaz E, Post $P$, Knorst $M$. Fatores de risco para internação por doença respiratória aguda em crianças até um ano de idade. Rev. Saude Publica 2007; 41(3):351-358.

31. Stiles WB. Research qualitative: evaluating the process and the product. In: Llewelyn S, Kennedy $\mathrm{P}$ editors. $\mathrm{H}$ andbook of clinical health psychology $\mathrm{New}$ York: Wiley; 2003. p. 477-499.

32. Turato ER. Tratado da metodologia da pesquisa clínico-qualitativa - construção teórico-epistemológica, discussão comparada e aplicação nas áreas de saúde e humanas. Petrópolis: Vozes; 2003.

33. Denzin NK, Lincoln YS. Collecting and interpreting qualitative materials. Thousand Oaks: Sage Publications; 1998.

34. Darbyshire P. Mother's experiences of their child's recovery in hospital and at home: A qualitative investigation. J Child Health Care 2003; 7(4):291-312.

35. Nakamura E, Santos JQ. Depressão infantil: abordagem antropológica. Rev. Saude Publica 2007; 41(1):53-60.

36. Nagai R, Lefèvre AMC, Lefèvre $F$, Steluti J, Teixeira LR, Zinn, LCS, Soares NS, Fischer FM. Conhecimentos e práticas de adolescentes na prevenção de acidentes de trabalho: estudo qualitativo. Rev. Saude Publica 2007; 41(3):404-411.

37. Almeida AFF, Hardy E. Vulnerabilidade de gênero para a paternidade em homens adolescentes. Rev. Saude Publica 2007; 41(4):565-572.

38. Sanchez ZVM, Oliveira LG, Nappo SA. Razões para o não uso de drogas ilícitas entre jovens em situação de risco. Rev. Saude Publica 2005; 39(4):599-605.
39. Fleith DS, Costa Júnior AL. M étodos de pesquisa em psicologia do desenvolvimento: o que é relevante considerar? In: Dessen MA, Costa-Júnior AL, organizadores. A ciência do desenvolvimento humano: tendências atuais e perspectivas futuras. Porto Alegre: Artmed; 2005. p. 264-278.

40. Breakweel GM. Research: Theory and method. In: Breakweel GM, Hammond S, Fife-Schow C, editors. Research methods in psychology. London: Sage Publications; 1995. p. 5-15.

41. Dessen MA, Costa Júnior $A L$, organizadores. A ciência do desenvolvimento humano: tendências atuais e perspectivas futuras. Porto Alegre: Artmed; 2005.

42. Magnusson D, Cairns RB. Developmental science: Toward a unified framework. In: Cairns RB, Elder JH, Costello EJ, editors. Developmental science. New York: Cambridge University Press; 1996. p. 7-30.

43. Gottlieb G. Developmental psychobiological theory. In: Cairns RB, Elder JH, Costello EJ, editors. Developmental Science. New York: Cambridge University Press; 1996. p. 63-76.

44. Bronfenbrenner U. Toward an experimental ecology of human development. American Psychologist 1977; 32:513-531.

45. Cairns RB, Elder GH, Costello EJ. Developmental science: a collaborative statement. Cairns RB, Elder $J \mathrm{H}$, Costello EJ, editors. Developmental science. New York: Cambridge University Press; 1996. p. 1-6.

46. Geert PV. Dynamic Systems approaches and modeling of developmental processes. In: Valsiner J, Connolly $\mathrm{K}$, editors. $\mathrm{H}$ andbook of developmental psychology. London: Sage Publications; 2003. p. 640-672.

47. Winegar LT. Developmental research and comparative perspective: applications to developmental science. In: Tudge J, Shanahan MJ, Valsiner J, editors. Comparisons in human development: Understanding time and context. New York: Cambridge University Press; 1997. p. 13-33.

48. Gottlieb G. Probabilistic Epigenesis of development. In: Valsiner J, Connolly K, editors. Handbook of developmental psychology. London: Sage Publications; 2003. p. 3-17.

49. Gauy FV, Costa Júnior AL. A natureza do desenvolvimento humano: contribuições das teorias biológicas. In: Dessen MA, Costa Júnior AL, organizadores. A ciência do desenvolvimento humano: tendências atuais e perspectivas futuras. Porto Alegre: Artmed; 2005. p. 53-70.

50. Sameroff AJ, Suomi SJ. Primates and persons: a comparative developmental understanding of social organization. In: Cairns RB, Elder GH, CostelIo EJ, editors. Developmental science. New York: Cambridge University Press; 1996. p. 97-120.

51. Dal-Farra RA, Prates EJ. A psicologia face aos novos progressos da genética humana. Psicol. Cienc. Prof. 2004; 24(1):94-107.

52. Plomin R. Behavioural genetics in the 21st century. Int J Behav Dev 2000; 24(1):30-34.

53. Aspesi CC, Dessen MA, Chagas JF. A ciência do desenvolvimento humano: uma perspectiva interdisciplinar. In: Dessen MA, Costa-Júnior AL, organizadores. A ciência do desenvolvimento humano: tendências atuais e perspectivas futuras. Porto Alegre: Artmed; 2005. p. 19-36. 
54. Elder GH. Human lives in changing societies: Life course and development insights. In: Cairns RB, Elder GH, Costello EJ, editors. Developmental science. New York: Cambridge University Press; 1996. p. 31-62.

55. Masten AS. Developmental psychopathology: Pathways to the future. Int J Behav Dev 2006; 30(1):47-54.

56. Cicchetti D. Developmental Psychopathology: reactions, reflections, projection. Dev Rev 1993; 13(4):471-502.

57. Shanahan MJ, Valsiner J, Gottlieb G. Developmental concepts across disciplines. In: Tudge J, Shanahan MJ, Valsiner J, editors. Comparisons in human development: Understanding time and context. $\mathrm{New}$ York: Cambridge University Press; 1997. p. 34-71.

58. Bronfenbrenner U. Ecological models of human development. In: Husten T, Postelethwaite TN, editors. International Encyclopedia of Education. N ew York: Elsevier Science; 1994. p. 1643-1647.

59. Bronfenbrenner U. Environments in developmental perspective: Theoretical and operational models. In: Friedman SL, Wachs TD, editors. M easuring environment across the life span: Emerging methods and concepts. Washington, D.C.: American Psychological Association; 1999. p. 3-28.

60. Bronfenbrenner U. A ecologia do desenvolvimento humano: experimentos naturais e planejados. Porto Alegre: Artes M édicas; 1996.

61. Narvaz M G, Koller SH. O modelo bioecológico do desenvolvimento humano. In: Koller SH, organizador. Ecologia do desenvolvimento humano. São Paulo: Casa do Psicólogo; 2004. p. 51-65.

62. Copetti F, Krebs RJ. As propriedades da pessoa na perspectiva do paradigma bioecológico. In: Koller $\mathrm{SH}$, organizador. Ecologia do desenvolvimento humano. São Paulo: Casa do Psicólogo; 2004. p. 67-89.

63. Bronfenbrenner $U$, Ceci SJ. Nature-Nurture reconceptualized in developmental perspective: A bioecological model. Psychol Rev 1994; 101(4):568-586.

64. Bronfenbrenner $U, M$ orris PA. The ecology of developmental processes. In: Damons W, Lerner RM, editors. Handbook of child psychology: Theoretical models of human development. New York: Wiley; 1998. p. 993-1028.

65. Bronfenbrenner U. Ecological system theory. In: Vasta R, editor. Six theories of child development. London: Jessica Kingsley; 1992. p. 187-243.

66. Dessen MA. Construindo uma ciência do desenvolvimento humano: passado, presente e futuro. In: Dessen MA, Costa Júnior $A L$, organizadores. A ciência do desenvolvimento humano: tendências atuais e perspectivas futuras. Porto Alegre: Artmed; 2005. p. 264-278.

67. Hepworth J. Public Health Psychology: a conceptual and practical framework. J Health Psychol 2004 9(1):41-54.

Artigo apresentado em 28/03/2008

Aprovado em 31/10/2008

Versão final apresentada em 19/01/2009 